\title{
Direct Cardiac Reprogramming - Converting Cardiac Fibroblasts to Cardiomyocytes -
}

\author{
Taketaro Sadahiro, MD, PhD
}

\begin{abstract}
Cardiovascular disease is the leading cause of death and disability worldwide. Despite advances in cardiovascular therapy, mortality in heart disease still remains high. Direct cardiac reprogramming is a promising approach for cardiac tissue repair involving in situ generation of new cardiomyocytes from endogenous cardiac fibroblasts. Although, initially, the reprogramming efficiency was low, several developments in reprogramming methods have improved the in vitro cardiac reprogramming efficiency. Subsequently, in vivo cardiac reprogramming has demonstrated improvement in cardiac function and fibrosis after myocardial infarction. Here, we review recent progress in cardiac reprogramming as a new technology for cardiac regeneration.
\end{abstract}

Key Words: Cardiomyocyte; Direct reprogramming; Fibroblast; Regeneration

C ardiovascular disease is the leading cause of death and disability worldwide. Because adult cardiac regenerative capacity is limited, dead cardiomyocytes (CM) are replaced by fibroblasts, leading to cardiac fibrosis, adverse ventricular remodeling, and significantly impaired heart function, which is associated with poor prognosis. ${ }^{1,2}$ Despite advances in cardiovascular pharmacotherapy and implantable medical devices, the mortality in heart failure still remains high. Although heart transplantation improves the prognosis, there are relatively few donors in Japan. Therefore, cardiac regenerative therapy is a rapidly emerging field aimed at repairing injured hearts. A promising approach for heart regeneration is the use of allogeneic pluripotent stem cell (PSC)-derived CM, such as embryonic stem cells and induced PSC (iPSC). With regard to PSC-derived CM, however, there are challenges to overcome before clinical application, such as low engraftment after cell transplantation, and the tumorigenic potential. ${ }^{3}$ In 2010, we discovered a novel approach to generating $\mathrm{CM}$ in an injured heart, which led to an approach for heart repair through direct cardiac reprogramming, where resident cardiac fibroblasts $(\mathrm{CF})$ are converted to induced CM-like cells (iCM) without reverting to PSC through transduction of cardiac-specific transcription factors. In this review, we summarize the advances in cardiac reprogramming for application in heart repair.

\section{Direct Reprogramming of Fibroblasts Into CM by Defined Factors \\ Discovery of Direct Cardiac Reprogramming by Gata4, Mef2c, and Tbx5}

The generation of specific cells from abundant and accessible cells holds great promise for regenerative medicine. In 2006, Takahashi and Yamanaka made the groundbreaking discovery that overexpression of 4 transcription factors, Oct4, Sox2, Klf4, and c-Myc (also known as the Yamanaka factors), in mouse fibroblasts induces a pluripotent state. ${ }^{4}$ The discovery of iPSC provided new approaches for generating desired cell types by overexpressing lineage-specific factors. ${ }^{5}$ The heart consists of CM and non-myocytes, such as endothelial cells, smooth muscle cells and CF. CM account for $30 \%$ of all cells in the heart. Recently developed techniques and genetic tools have been able to clarify the distribution of major non-myocyte cardiac cell types, with CF constituting $20 \%$ of the non-myocytes in the heart. ${ }^{6}$ Moreover, resident $\mathrm{CF}$ can be activated and increased in response to various types of cardiac injury, contributing to scar formation upon cardiac damage and poor prognosis. ${ }^{7-9}$ Thus, CF are a source of abundant and accessible nonmyocytes in an injured heart, and represent a potential source of CM for cardiac regeneration.

We hypothesized that overexpression of key developmental cardiac regulators could, in mouse fibroblasts, induce a cardiac state. To test this hypothesis, we first generated $\alpha$-myosin heavy chain promoter-driven enhanced green fluorescent protein ( $\alpha$ MHC-GFP) transgenic mice, in which cells with activated cardiac programs express GFP. Following retroviral expression of candidate genes in $\mathrm{CF}$, we found that a combination of 3 transcription factors (Gata4, Mef2c, and Tbx5; GMT), could directly convert $\mathrm{CF}$ into iCM. ${ }^{10}$ Lineage-tracing experiments confirmed that cardiac progenitor genes were not activated during the reprogramming process, suggesting that fibroblasts were converted directly into iCM without passing through a progenitor cell state. iCM had similar morphology and function to those of endogenous CM, such as wellorganized sarcomeric structures, similar global gene expression profiles, and spontaneous beating.

It is important to understand how a transcription factor

Received October 3, 2019; accepted October 3, 2019; J-STAGE Advance Publication released online November 12, 2019

Department of Cardiology, Faculty of Medicine, University of Tsukuba, Tsukuba, Japan

Mailing address: Taketaro Sadahiro, MD, PhD, Department of Cardiology, Faculty of Medicine, University of Tsukuba, 1-1-1

Tennoudai, Tsukuba 305-8575, Japan. E-mail: taketarosadahiro@md.tsukuba.ac.jp

ISSN-2434-0790 All rights are reserved to the Japanese Circulation Society. For permissions, please e-mail: cr@j-circ.or.jp 

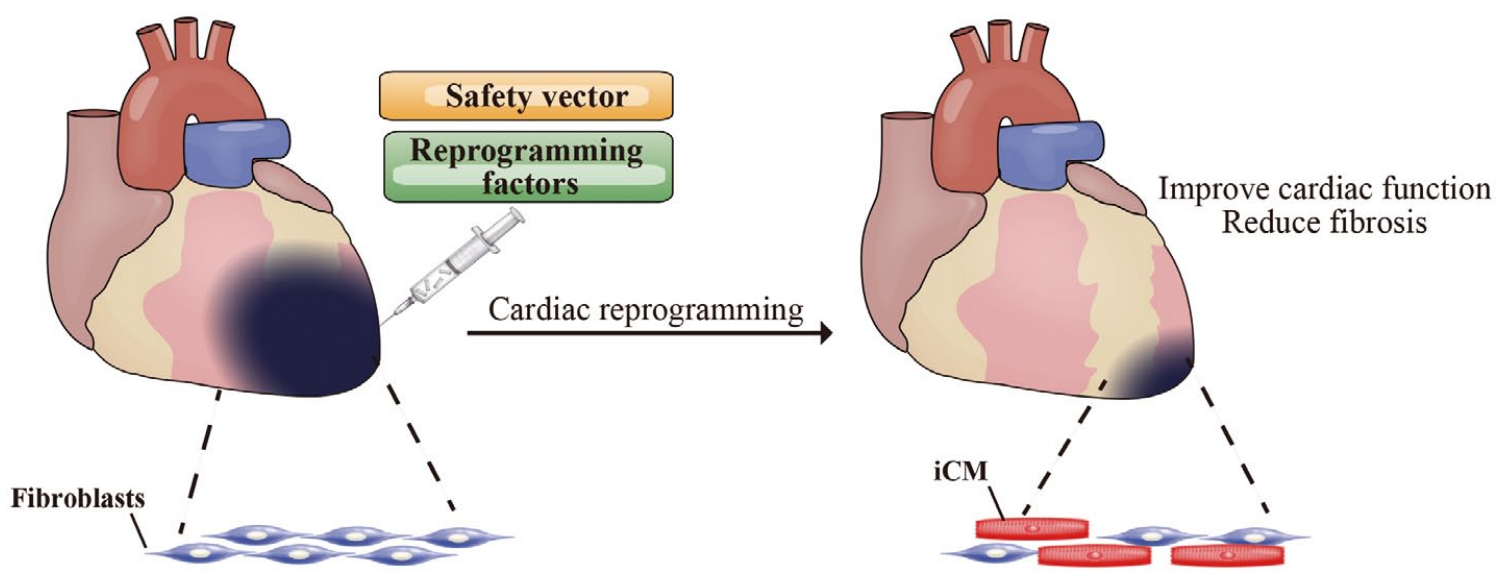

Figure. Challenges to generating cardiomyocytes for cardiac regeneration. Myocardial fibrosis was resolved and cardiac function improved after cardiac direct reprogramming. iCM, induced cardiomyocyte-like cells.

could directly control the reprogramming process; for example, promoter and enhancer elements of cardiacspecific genes would be accessible for transcription factor binding in fibroblasts. GMT promotes expression of genes associated with CM while simultaneously repressing the fibroblast gene program. Recent integrated sequence analysis showed that Mef2c plays an essential role during cardiac reprogramming. ${ }^{\mathbf{1 1}, 12}$ Mechanistically, Mef2c and Tbx 5 independently promote chromatin remodeling at previously inaccessible sites and induce binding of the additional reprogramming factors. Moreover, GMT rapidly silences fibroblast enhancers and synergistically activates cardiac enhancers highly enriched with Mef2c binding sites. Thus, the 3 components of GMT independently, and in collaboration, initiate cell fate conversions.

\section{Development of Cardiac Reprogramming}

Optimization of reprogramming cocktails and modification of signaling pathways and epigenetic alterations represent potential strategies to promote reprogramming efficiency and maturation. Song et al investigated the optimal combination of 6 conserved cardiac-lineage transcription factors, including GMT, and demonstrated that GMT factors plus Hand 2 resulted in more efficient reprogramming of CF into beating $\mathrm{iCM}$ as compared with GMT alone. ${ }^{13}$ Alternatively, combinations of microRNA (miR) could directly convert CF into iCM. miR play an important role in cell fate and embryonic cardiac development by regulating numerous genes related to signaling pathways, transcription factors, and epigenetic regulators. ${ }^{\mathbf{1 4}, 15}$ Jayawardena et al demonstrated how a combination of muscle-specific miR (miR-1, miR-133, miR-208, and miR-499) with CF could be used to successfully generate functional iCM. ${ }^{16,17} \mathrm{miR}$ bind to the $3^{\prime}$ untranslated region of their target mRNA to repress protein translation. Muraoka et al found that miR133 plus GMT improves cardiac reprogramming by miR133 by directly targeting Snail, a master regulator of the epithelial-to-mesenchymal transition. ${ }^{18}$ miR-133 plus GMT also shortened the time required to induce beating iCM, to one-third of that using GMT alone. They also found that fibroblast signatures are a barrier to efficient cardiac reprogramming and need to be silenced to allow successful reprogramming. ${ }^{18}$ Subsequently, many groups demonstrated that inhibition of pro-fibrotic signaling pathways promoted reprogramming efficiency. The transforming growth factor (TGF)- $\beta$, Wnt and Roh-kinase (ROCK) pathways play a key role in fibroblast activation. Ifkovits et al showed that inhibition of TGF- $\beta$ signaling increased cardiac reprogramming by silencing fibroblast signatures. ${ }^{19}$ Mohamed et al confirmed that inhibition of TGF- $\beta$ and Wnt signaling enhanced cardiac-reprogramming efficiency and maturation. ${ }^{20}$ Moreover, Zhao et al showed that inhibition of TGF- $\beta$ and ROCK pathways convert fibroblasts into iCM with an efficiency of up to $60 \%{ }^{21}$ Inhibition of pro-fibrotic pathways promotes cardiac reprogramming, although activating the Akt pathway enhanced generation of mature iCM. Zhou et al found that activation of the Aktl pathway promoted reprogramming efficiency and $\mathrm{iCM}$ maturation. ${ }^{22}$ Yamakawa et al found that serum-free culture medium containing cariogenic growth factors, such as fibroblast growth factor (FGF)2, FGF10, and vascular endothelial growth factor (VEGF), greatly enhanced the generation of beating mature iCM by activating the p38 Mitogenactivated Protein Kinase (MAPK) pathway and the phosphoinositide 3-kinase/Akt pathway. ${ }^{23}$ This suggested that modifications of signaling pathways may alter the efficiency of direct cardiac reprogramming.

The selection of the starting cell type is another key choice in successful direct reprogramming. The starting fibroblast type may ease or impede the reprogramming process, depending on the cellular context upon which the GMT act. Mouse embryonic fibroblasts (MEF) are easily converted into fully reprogrammed $\mathrm{iCM}$, whereas cardiac reprogramming of more differentiated fibroblasts, such as CF and adult tail-tip fibroblasts (TTF), is difficult and has been achieved only in immature iCM. ${ }^{5}$ Recently, we found that inflammation acts as a barrier to direct cardiac reprogramming, particularly in aged TTF. ${ }^{24}$ High-throughput screening of 8,400 chemical libraries for compounds associated with cardiac reprogramming indicated that diclofenac sodium, a non-steroidal anti-inflammatory drug, promotes reprogramming efficiency and maturation in adult TTF, but not in MEF, in combination with GMT. Mechanistically, cyclooxygenase-2 (COX-2) is strongly expressed in adult 
TTF, as compared with MEF, in an age-dependent manner. Diclofenac enhances cardiac reprogramming by inhibiting signaling associated with $\mathrm{COX}-2$, prostaglandin $\mathrm{E} 2$ receptor 4 , cyclic AMP/protein kinase A, and interleukin- $1 \beta$, and by silencing inflammatory and fibroblast programs, which are activated in adult TTF. Thus, a better understanding of the molecular mechanisms will result in successful cardiac reprogramming, and promote the development of novel methods for cardiac reprogramming.

\section{In Vivo Direct Cardiac Reprogramming and Heart Repair}

The goal of cardiac regenerative therapy is to provide a functional $\mathrm{CM}$ to replace the lost myocardium in vivo. Interestingly, in vivo conditions have the potential to promote greater reprogramming efficiency and maturity. In vivo direct reprogramming in mice was reported following retroviral or lentiviral delivery of reprogramming factors following acute myocardial infarction (MI). ${ }^{13,17,25,26}$ The lineage-tracing approach demonstrated that these newly generated $\mathrm{iCM}$ were derived from resident $\mathrm{CF}$ and formed gap junctions with endogenous CM. ${ }^{13}$ In vivo reprogramming improved cardiac systolic function, and scar areas were significantly smaller at 8 weeks after MI in the in vivo reprogramming group than in the control group. Moreover, on genome-wide transcriptional profiling, in vivo iCM had a closer similarity to bona fide CM than in vitro iCM.27 This indicated that the unidentified factors in the native microenvironment in vivo, including extracellular matrix, secreted proteins, and that electromechanical stimulation might enforce direct cardiac reprogramming. ${ }^{28}$

To translate this new therapeutic approach into clinical use, the development of a safe delivery vector is required to avoid insertional mutagenesis. Although retroviral vectors selectively infect non-CM, mainly $\mathrm{CF}$, in infarcted hearts, these vectors risk the integration of the delivered gene sequences into host chromosomes. Recently, Miyamoto et al developed a Sendai virus vector expressing GMT (SeV-GMT) and demonstrated its use for successful in vivo direct cardiac reprogramming after $\mathrm{MI} .^{29}$ In contrast to retroviral vectors, $\mathrm{SeV}$ does not integrate into the host genome. Moreover, this new integration-free SeV-GMT enhanced in vivo cardiac reprogramming compared with conventional retrovirus vectors. Although further refinements are needed, in vivo cardiac reprogramming with $\mathrm{SeV}$ might be a promising approach for cardiac regeneration in the future.

\section{Challenges for Clinical Translation in Direct Cardiac Reprogramming}

Despite substantial progress in this field, several challenges remain to be overcome prior to clinical application. Although in vivo cardiac reprogramming has allowed impressive improvements in cardiac function and fibrosis after acute $\mathrm{MI},{ }^{13,20,25,26,29}$ it remains to be determined whether in vivo cardiac reprogramming could be applied to chronic heart failure, such as chronic MI. It is technically difficult to inject viral vectors into the infarcted hearts due to thin scar tissue and the re-thoracotomy procedure. Moreover, a gene delivery system to resident $\mathrm{CF}$ remains elusive, which may hinder research into cardiac reprogramming in chronic heart failure. Cardiac reprogramming in human $\mathrm{CF}$ also remains inefficient. ${ }^{20,30-32}$ Although critical factors have been identified in mouse cardiac reprogramming, GMT is insufficient to achieve human cardiac reprogramming and additional reprogramming factors are needed. Human iCM generated with GMT plus additional factors have been shown to express cardiac genes, sarcomeric structures, and $\mathrm{Ca}^{2+}$ activity in a prolonged culture, but they did not beat spontaneously. Recently, single-cell RNA sequencing showed that human cardiac reprogramming progresses at a much slower rate toward the iCM fate than does murine reprogramming. ${ }^{33}$ It was also shown that inflammation is critical for human iCM reprogramming. ${ }^{33}$ Although the inflammatory response was identified as a molecular barrier to cardiac reprogramming in mouse cells, inhibition of inflammatory signaling led to a profound reduction in human $\mathrm{iCM}$ induction through epigenetic changes. ${ }^{33}$ The mechanism underlying the opposite roles of inflammation in mouse and human cells in cardiac reprogramming is also still to be clarified. Despite the challenges that still remain, we believe that new technologies and continuing research into cardiac reprogramming may provide new insights to rapidly overcome these hurdles, and that this promising approach can be clinically applied in the near future (Figure).

\section{Acknowledgments}

T.S. was supported by research grants from JSPS (18K08114), the Nakatomi Foundation, and the Mochida Memorial Foundation for Medical and Pharmaceutical Research.

\section{Disclosures}

T.S. declares no conflicts of interest.

\section{References}

1. Furtado MB, Nim HT, Boyd SE, Rosenthal NA. View from the heart: Cardiac fibroblasts in development, scarring and regeneration. Development 2016; 143: 387-397.

2. Fan D, Takawale A, Lee J, Kassiri Z. Cardiac fibroblasts, fibrosis and extracellular matrix remodeling in heart disease. Fibrogenesis Tissue Repair 2012; 5: 15.

3. Yoshida Y, Yamanaka S. Induced pluripotent stem cells 10 years later: For cardiac applications. Circ Res 2017; 120: 1958-1968.

4. Takahashi K, Yamanaka S. Induction of pluripotent stem cells from mouse embryonic and adult fibroblast cultures by defined factors. Cell 2006; 126: 663-676.

5. Sadahiro T, Yamanaka S, Ieda M. Direct cardiac reprogramming: Progress and challenges in basic biology and clinical applications. Circ Res 2015; 116: 1378-1391.

6. Pinto AR, Ilinykh A, Ivey MJ, Kuwabara JT, D’Antoni ML, Debuque R, et al. Revisiting cardiac cellular composition. Circ Res 2016; 118: 400-409.

7. Sadahiro T, Kohsaka S, Okuda S, Inohara T, Shiraishi Y, Kohno T, et al. MRI and serum high-sensitivity $C$ reactive protein predict long-term mortality in non-ischaemic cardiomyopathy. Open Heart 2015; 2: e000298.

8. Travers JG, Kamal FA, Robbins J, Yutzey KE, Blaxall BC. Cardiac fibrosis: The fibroblast awakens. Circ Res 2016; 118: $1021-1040$.

9. Fu X, Khalil H, Kanisicak O, Boyer JG, Vagnozzi RJ, Maliken $\mathrm{BD}$, et al. Specialized fibroblast differentiated states underlie scar formation in the infarcted mouse heart. J Clin Invest 2018; 128: 2127-2143.

10. Ieda M, Fu JD, Delgado-Olguin P, Vedantham V, Hayashi Y, Bruneau BG, et al. Direct reprogramming of fibroblasts into functional cardiomyocytes by defined factors. Cell 2010; 142: 375-386.

11. Hashimoto H, Wang Z, Garry GA, Malladi VS, Botten GA, Ye $\mathrm{W}$, et al. Cardiac reprogramming factors synergistically activate genome-wide cardiogenic stage-specific enhancers. Cell Stem Cell 2019; 25: 69-86.e65.

12. Stone NR, Gifford CA, Thomas R, Pratt KJB, Samse-Knapp K, Mohamed TMA, et al. Context-specific transcription factor functions regulate epigenomic and transcriptional dynamics 
during cardiac reprogramming. Cell Stem Cell 2019; 25: 87-102. e109.

13. Song K, Nam YJ, Luo X, Qi X, Tan W, Huang GN, et al. Heart repair by reprogramming non-myocytes with cardiac transcription factors. Nature 2012; 485: 599-604.

14. Cordes KR, Srivastava D, Ivey KN. MicroRNAs in cardiac development. Pediatr Cardiol 2010; 31: 349-356.

15. Hammond SM. An overview of microRNAs. Adv Drug Deliv Rev 2015; 87: 3-14.

16. Jayawardena TM, Egemnazarov B, Finch EA, Zhang L, Payne JA, Pandya K, et al. MicroRNA-mediated in vitro and in vivo direct reprogramming of cardiac fibroblasts to cardiomyocytes. Circ Res 2012; 110: 1465-1473.

17. Jayawardena TM, Finch EA, Zhang L, Zhang H, Hodgkinson $\mathrm{C}$, Pratt RE, et al. MicroRNA induced cardiac reprogramming in vivo: Evidence for mature cardiac myocytes and improved cardiac function. Circ Res 2015; 116: 418-424.

18. Muraoka N, Yamakawa H, Miyamoto K, Sadahiro T, Umei T, Isomi M, et al. MiR-133 promotes cardiac reprogramming by directly repressing Snail and silencing fibroblast signatures. EMBO J 2014; 33: $1565-1581$.

19. Ifkovits JL, Addis RC, Epstein JA, Gearhart JD. Inhibition of TGFbeta signaling increases direct conversion of fibroblasts to induced cardiomyocytes. PLoS One 2014; 9: e89678.

20. Mohamed TM, Stone NR, Berry EC, Radzinsky E, Huang Y, Pratt K, et al. Chemical enhancement of in vitro and in vivo direct cardiac reprogramming. Circulation 2017; 135: 978-995.

21. Zhao Y, Londono P, Cao Y, Sharpe EJ, Proenza C, O'Rourke R, et al. High-efficiency reprogramming of fibroblasts into cardiomyocytes requires suppression of pro-fibrotic signalling. Nat Commun 2015; 6: 8243.

22. Zhou H, Dickson ME, Kim MS, Bassel-Duby R, Olson EN. Akt1/protein kinase B enhances transcriptional reprogramming of fibroblasts to functional cardiomyocytes. Proc Natl Acad Sci USA 2015; 112: 11864-11869.

23. Yamakawa H, Muraoka N, Miyamoto K, Sadahiro T, Isomi M, Haginiwa S, et al. Fibroblast growth factors and vascular endo- thelial growth factor promote cardiac reprogramming under defined conditions. Stem Cell Rep 2015; 5: 1128-1142.

24. Muraoka N, Nara K, Tamura F, Kojima H, Yamakawa H, Sadahiro T, et al. Role of cyclooxygenase-2-mediated prostaglandin E2-prostaglandin E receptor 4 signaling in cardiac reprogramming. Nat Commun 2019; 10: 674.

25. Qian L, Huang Y, Spencer CI, Foley A, Vedantham V, Liu L, et al. In vivo reprogramming of murine cardiac fibroblasts into induced cardiomyocytes. Nature 2012; 485: 593-598.

26. Inagawa K, Miyamoto K, Yamakawa H, Muraoka N, Sadahiro $\mathrm{T}$, Umei $\mathrm{T}$, et al. Induction of cardiomyocyte-like cells in infarct hearts by gene transfer of Gata4, Mef2c, and Tbx5. Circ Res 2012; 111: $1147-1156$.

27. Cahan P, Li H, Morris SA, Lummertz da Rocha E, Daley GQ, Collins JJ. CellNet: Network biology applied to stem cell engineering. Cell 2014; 158: 903-915.

28. Ronaldson-Bouchard K, Ma SP, Yeager K, Chen T, Song L, Sirabella D, et al. Advanced maturation of human cardiac tissue grown from pluripotent stem cells. Nature 2018; 556: 239-243.

29. Miyamoto K, Akiyama M, Tamura F, Isomi M, Yamakawa H, Sadahiro T, et al. Direct in vivo reprogramming with Sendai virus vectors improves cardiac function after myocardial infarction. Cell Stem Cell 2018; 22: 91-103.e105.

30. Nam YJ, Song K, Luo X, Daniel E, Lambeth K, West K, et al. Reprogramming of human fibroblasts toward a cardiac fate. Proc Natl Acad Sci USA 2013; 110: 5588-5593.

31. Wada R, Muraoka N, Inagawa K, Yamakawa H, Miyamoto K, Sadahiro T, et al. Induction of human cardiomyocyte-like cells from fibroblasts by defined factors. Proc Natl Acad Sci USA 2013; 110: $12667-12672$.

32. Fu JD, Stone NR, Liu L, Spencer CI, Qian L, Hayashi Y, et al. Direct reprogramming of human fibroblasts toward a cardiomyocyte-like state. Stem Cell Rep 2013; 1: 235-247.

33. Zhou Y, Liu Z, Welch JD, Gao X, Wang L, Garbutt T, et al. Single-cell transcriptomic analyses of cell fate during human cardiac reprogramming. Cell Stem Cell 2019; 25: 149-164.e149. 